\title{
CAIX aptamer-functionalized targeted nanobubbles for ultrasound molecular imaging of various tumors
}

This article was published in the following Dove Press journal: International Journal of Nanomedicine

\author{
Lianhua Zhu' \\ Luofu Wang ${ }^{2}$ \\ Yu Liu' \\ Dan Xu' \\ Kejing Fang' \\ Yanli Guo' \\ 'Department of Ultrasound, \\ Southwest Hospital, Third Military \\ Medical University (Army Medical \\ University), Shapingba District, \\ Chongqing, China; ${ }^{2}$ Department \\ of Urology, Daping Hospital, Third \\ Military Medical University (Army \\ Medical University), Yuzhong District, \\ Chongqing, China
}

\begin{abstract}
Purpose: Targeted nanobubbles can penetrate the tumor vasculature and achieve ultrasound molecular imaging (USMI) of tumor parenchymal cells. However, most targeted nanobubbles only achieve USMI of tumor parenchymal cells from one organ, and their distribution, loading ability, and binding ability in tumors are not clear. Therefore, targeted nanobubbles loaded with carbonic anhydrase IX (CAIX) aptamer were fabricated for USMI of various tumors, and the morphological basis of USMI with targeted nanobubbles was investigated.
\end{abstract}

Materials and methods: The specificity of CAIX aptamer at the cellular level was measured by immunofluorescence and flow cytometry. Targeted nanobubbles loaded with CAIX aptamer were prepared by a maleimide-thiol coupling reaction, and their binding ability to CAIX-positive tumor cells was analyzed in vitro. USMI of targeted and non-targeted nanobubbles was performed in tumor-bearing nude mice. The distribution, loading ability, and binding ability of targeted nanobubbles in xenograft tumor tissues were demonstrated by immunofluorescence.

Results: CAIX aptamer could specifically bind to CAIX-positive 786-O and Hela cells, rather than CAIX-negative BxPC-3 cells. Targeted nanobubbles loaded with CAIX aptamer had the advantages of small size, uniform distribution, regular shape, and high safety, and they could specifically accumulate around 786-O and Hela cells, while not binding to BxPC-3 cells in vitro. Targeted nanobubbles had significantly higher peak intensity and larger area under the curve than non-targeted nanobubbles in 786-O and Hela xenograft tumor tissues, while there was no significant difference in the imaging effects of targeted and non-targeted nanobubbles in BxPC-3 xenograft tumor tissues. Immunofluorescence demonstrated targeted nanobubbles could still load CAIX aptamer after penetrating the tumor vasculature and specifically binding to CAIX-positive tumor cells in xenograft tumor tissues.

Conclusion: Targeted nanobubbles loaded with CAIX aptamer have a good imaging effect in USMI of tumor parenchymal cells, and can improve the accuracy of early diagnosis of malignant tumors from various organs.

Keywords: targeted ultrasound contrast agents, molecular imaging, malignant tumors, aptamers, morphological basis

\section{Introduction}

The ultrasound contrast agents (UCAs) that are currently used in the clinic can only achieve perfusion imaging of tumor vasculature. Due to the lack of tumor specificity, they cannot specifically accumulate in tumor tissues and thus are not able to achieve ultrasound molecular imaging (USMI) of malignant tumors. ${ }^{1,2}$ Targeted UCAs can be constructed by coupling specific ligands against tumor cells with the surfaces of UCAs, which can specifically accumulate in tumor tissues and be used for USMI of malignant 
tumors. Targeted UCAs are mostly on the micron scale, and their binding targets are mainly membrane proteins of tumor vascular endothelial cells, such as vascular endothelial growth factor receptor-2, endothelin, nucleolin, and so on. ${ }^{3-6}$ Therefore, they only specifically bind to tumor vasculature and achieve USMI of tumor blood pool. It is a challenge for targeted UCAs to penetrate the tumor vasculature and achieve extravascular USMI of malignant tumors.

Nanoscale UCAs with small particle size, strong penetration, and long imaging time can aggregate in the extravascular spaces via the enhanced permeability and retention (EPR) effect. $^{7}$ Extravascular targeted ligands on the surfaces of targeted nanoscale UCAs can further enhance the accumulation of nanoscale UCAs in tumor tissues. After intravenous injection, targeted nanoscale UCAs can specifically bind to tumor parenchyma cells, achieve extravascular USMI of malignant tumors, and find the changes in protein expression on the cell membranes of tumor parenchyma cells in vivo. ${ }^{8,9}$ Nanobubbles with a lipid outer shell are a novel type of nanoscale UCAs. ${ }^{10}$ Compared with other shell materials, nanobubbles with a lipid shell have a good imaging effect and high biocompatibility, and have been widely applied in USMI. ${ }^{1-14}$ However, current studies about USMI of targeted lipid nanobubbles have two challenges: 1) targeted nanobubbles are mainly used for USMI of tumor parenchyma cells from one organ, such as targeted nanobubbles carrying anti-prostate specific membrane antigen aptamer mainly achieve USMI of tumor parenchymal cells in prostate cancer. ${ }^{15}$ Although they can achieve intravascular USMI in non-prostatic tumors, USMI of tumor parenchymal cells in non-prostatic tumors cannot be achieved. ${ }^{16,17}$ 2) Most studies only focus on the imaging effect of targeted nanobubbles and have not yet studied their distribution, loading ability, and specific binding ability in vivo.

Carbonic anhydrase IX (CAIX) plays an important role in the growth and metastasis of numerous tumors (including renal cancer, cervical cancer, colon cancer, prostate cancer, breast cancer, head and neck tumors, and so on), and has been used for the diagnosis, treatment, and prognosis of malignant tumors. ${ }^{18-23}$ Targeted ligands against CAIX include monoclonal antibodies, nanobodies, and affibodies, as well as polypeptides that we studied earlier. ${ }^{18,22,24,25}$ Aptamers are single-stranded DNA or RNA oligonucleotides that specifically bind to biological macromolecules or cells through a specific spatial structure. Compared with other ligands, aptamers have several attractive features, including diverse structure, high specificity, high stability, rapid tissue penetration, no immunogenicity, and easy synthesis and modification. ${ }^{26-29}$ Molecular probes loaded with specific aptamers can specifically gather in target tissues and achieve molecular imaging of target tissues. ${ }^{15,30}$

This study prepared targeted lipid nanobubbles loaded with CAIX aptamer, explored their specific binding ability, and targeted imaging ability in various tumors, and further investigated the morphological basis of USMI in vivo. The aim was not only to provide a novel UCA with small size, safety, and high specificity for USMI, but also to provide the experimental basis and methods for targeted nanobubbles to achieve USMI of tumor parenchyma cells from various organs.

\section{Materials and methods Screening and sequencing of CAIX aptamer}

A single-stranded DNA strand against CAIX was screened by the systematic evolution of ligands by exponential enrichment. Magnetic beads (Thermo Fisher Scientific, Waltham, MA, USA) were incubated with CAIX (Sino Biological Inc., Beijing, China) for 30 minutes to obtain the magnetic beads functionalized with CAIX. An initial single-stranded DNA library $(20 \mu \mathrm{M})$ was heated at $95^{\circ} \mathrm{C}$ for 10 minutes and cooled immediately. Magnetic beads were incubated with the renatured DNA library for 30 minutes, and the single-stranded DNA strands unbound to magnetic beads were obtained. The single-stranded DNA strands were incubated with the magnetic beads functionalized with CAIX for 2 hours, and the single-stranded DNA strands bound to CAIX were obtained. The single-stranded DNA strands were PCR-amplified using phosphorylation-modified primers and then used as the new library for the next round of screening. After 10 rounds of screening, a single-stranded DNA strand specifically bound to CAIX was obtained and sequenced.

\section{Cell culture}

Human renal cell carcinoma 786-O cells, human cervical cancer Hela cells and human pancreatic cancer BxPC-3 cells used in the experiment were all from American Type Culture Collection (Manassas, VA, USA). All tumor cells were cultured in Roswell Park Memorial Institute 1640 supplemented with $10 \%$ FBS and $100 \mathrm{IU} / \mathrm{mL}$ penicillinstreptomycin (Thermo Fisher Scientific), and maintained at $37^{\circ} \mathrm{C}$ in an incubator containing $5 \% \mathrm{CO}_{2}$.

\section{Synthesis and evaluation of CAIX aptamer}

CAIX aptamer modified with a thiol group at the $3^{\prime}$ terminus and 6-carboxyfluorescein (6-FAM) at the $5^{\prime}$ terminus was 
synthesized by chemical synthesis. A nonsense aptamer of the same sequence length was synthesized and used as a negative control (Sangon Biotech (Shanghai) Co., Ltd., Shanghai, China). The binding ability of CAIX aptamer to CAIX-positive 786-O and Hela cells was measured by immunofluorescence, and CAIX-negative BxPC-3 cells were used as a control. The tumor cells were incubated with $2.5 \mu \mathrm{M}$ CAIX aptamer or nonsense aptamer at $37^{\circ} \mathrm{C}$ for 60 minutes and stained with DAPI (Beyotime Institute of Biotechnology, Shanghai, China). The binding ability of these two aptamers to the tumor cells was observed using a Zeiss 780 confocal laser scanning microscopy (CLSM; Carl Zeiss AG, Oberkochen, Germany). In addition, the tumor cells $\left(1 \times 10^{5} / \mathrm{mL}\right)$ were incubated with $2.5 \mu \mathrm{M}$ CAIX aptamer, nonsense aptamer, or PBS for 60 minutes, and the number of fluorescence-labeled tumor cells was measured using a FACSCalibur flow cytometer (BD Biosciences, San Jose, CA, USA) to quantitatively analyze the binding ability of these two aptamers to the tumor cells.

\section{Preparation of targeted nanobubbles}

The lipid components, including 1,2-dipalmitoyl-snglycero-3-phosphoethanolamine (Corden Pharma, Liestal, Switzerland), maleimide-modified 1,2-distearoyl-sn-glycero3-phosphoethanolamine (NANOCS, Boston, MA, USA), and 1,2-dipalmitoyl-sn-glycero-3-phosphocholine (Avanti Polar Lipids, Alabaster, AL, USA), were fully dissolved in a solution containing glycerin and PBS. Perfluoropropane gas (Research Institute of Physical and Chemical Engineering of Nuclear Industry, Tianjin, China) replaced the air in the bottle, and the bottle was shaken for 90 seconds in an ST-B series amalgamator (AT\&M Biomaterials Co., Beijing, China). The obtained suspension was centrifuged at 1,300 rpm for 3 minutes to remove the bottom lipid material that did not form bubbles, and the supernatant mixture including nanobubbles and microbubbles was obtained. The supernatant mixture was continuously centrifuged at $300 \mathrm{rpm}$ for 3 minutes to remove supernatant microbubbles and to obtain blank nanobubbles. A concentration of $10 \mathrm{mM}$ EDTA (Beyotime Institute of Biotechnology), $10 \mathrm{mM}$ Tris (2-carboxyethyl) phosphine hydrochloride (Thermo Fisher Scientific), and $2 \mathrm{mM} \mathrm{CAIX} \mathrm{aptamer} \mathrm{were} \mathrm{reacted} \mathrm{at} 37^{\circ} \mathrm{C}$ for 1 hour. Blank nanobubbles were then added and incubated for 2 hours. ${ }^{31}$ After the completion of coupling reaction, targeted nanobubbles loaded with CAIX aptamer were obtained and washed three times with cold PBS by centrifugation flotation at $300 \mathrm{rpm}$ for 3 minutes. Targeted nanobubbles floated on the top of the suspension after centrifugation, and the bottom solution was removed. Non-targeted nanobubbles loaded with nonsense aptamer were prepared by the same method and used as a control. To further evaluate the ability of targeted nanobubbles to load CAIX aptamer, CAIX aptamer modified with 6-FAM was loaded on targeted nanobubbles and the lipid membranes of targeted nanobubbles were labeled with 1,1'-dioctadecyl-3,3,3',3'-tetramethylindocarbocyanine perchlorate (DiI; Beyotime Institute of Biotechnology) dye, and the fluorescence distribution on the surfaces of targeted nanobubbles was observed under CLSM.

\section{Characteristics of targeted nanobubbles}

A hemocytometer was used to measure the concentration of targeted and non-targeted nanobubbles. The particle size and dispersion degree of targeted and non-targeted nanobubbles were compared using a Malvern Zetasizer Nano ZS90 detector (Malvern Instruments Inc., Worcestershire, UK). The distribution and morphology of targeted nanobubbles were observed using a JEM-1400 transmission electron microscope (JEOL, Tokyo, Japan) after negative staining with phosphotungstic acid. A Cell Counting Kit-8 (CCK-8; Beyotime Institute of Biotechnology) assay was used to assess the cytotoxicity of lipid material to 786-O cells. A $50 \mathrm{~mm}$ linear broadband probe and an IU22 ultrasound diagnosis apparatus (Philips, Amsterdam, the Netherlands) were used to perform ultrasound imaging of targeted and non-targeted nanobubbles at various concentrations $\left(1 \times 10^{8} / \mathrm{mL}, 5 \times 10^{7} / \mathrm{mL}, 2 \times 10^{7} / \mathrm{mL}, 1 \times 10^{7} / \mathrm{mL}\right.$, and $\left.5 \times 10^{6} / \mathrm{mL}\right)$ in vitro. Ultrasound images were collected under contrast-enhanced ultrasound (CEUS) mode (the mechanical index was 0.12 , and the frequency was 5-12 MHz). The imaging intensity of targeted and non-targeted nanobubbles in vitro was analyzed using Qlab 8.1 quantitative analysis software (Philips).

\section{Binding ability of targeted nanobubbles in vitro}

After culturing overnight, 786-O, Hela and BxPC-3 cells were each separated into three groups and incubated with DiI-labeled nanobubbles. One group was incubated with targeted nanobubbles $\left(1.5 \times 10^{8} / \mathrm{mL}\right)$, one group was incubated with non-targeted nanobubbles $\left(1.5 \times 10^{8} / \mathrm{mL}\right)$, and another group was reacted with $100 \mu \mathrm{M}$ CAIX aptamer for 60 minutes before incubation with targeted nanobubbles $\left(1.5 \times 10^{8} / \mathrm{mL}\right)$. After incubation at $37^{\circ} \mathrm{C}$ for 1 hour, unbound nanobubbles were removed, and the binding ability of targeted and nontargeted nanobubbles to the tumor cells in vitro was observed under CLSM. 


\section{Imaging effect of targeted nanobubbles in vivo}

The animal protocol in this study was approved by the Laboratory Animal Welfare and Ethics Committee of the Third Military Medical University, and the animals were cared for according to the guidelines of the Third Military Medical University for Animal Welfare. 786-O, Hela and BxPC-3 cells $\left(1 \times 10^{7} / \mathrm{mL}\right)$ were subcutaneously injected into the back of 4- to 6-week-old BALB/c-nu nude mice (HFK Bioscience Co., Beijing, China). After the length of xenograft tumor tissues reached $0.8-1.2 \mathrm{~cm}$, eight tumor-bearing nude mice of each tumor cell type were used for ultrasound imaging. After intraperitoneal injection of $1 \%$ pentobarbital sodium, a $50 \mathrm{~mm}$ linear broadband probe and an IU22 ultrasound diagnosis apparatus were used to display the maximum section of xenograft tumor tissues, and then the probe was fixed. Targeted or non-targeted nanobubbles $\left(1.5 \times 10^{8} / \mathrm{mL}\right)$ were randomly injected into each tumor-bearing nude mouse through the retroorbital venous sinus, and dynamic images were collected under CEUS mode (the mechanical index was 0.12 , and the frequency was $5-12 \mathrm{MHz}$ ). The "manual flash" technique was used to destruct residual nanobubbles before injection of another type of nanobubble. The injection interval between targeted and non-targeted nanobubbles was longer than an hour, which guaranteed the imaging effects would not interfere with each other. The imaging intensity of nanobubbles at different times was obtained using Qlab 8.1 quantitative analysis software, and peak time, peak intensity, and area under the curve of targeted and non-targeted nanobubbles in xenograft tumor tissues were compared.

To further evaluate the aggregation ability of targeted nanobubbles in tumor-bearing nude mice, fluorescence imaging of live small animals was performed. After anesthesia with isoflurane, 1, 1'-dioctadecyl-3,3,3', $3^{\prime}$ tetramethylindotricarbocyanine iodide (DiR; Thermo Fisher Scientific)-labeled targeted nanobubbles $\left(5 \times 10^{8} / \mathrm{mL}\right)$ were injected into each tumor-bearing nude mouse through the retroorbital venous sinus. The variations in fluorescence intensity of xenograft tumor tissues at different times ( 0 minute, 1 minute, 5 minutes, and 10 minutes) were compared using an IVIS Spectrum living animal imaging system (PerkinElmer Inc., Waltham, MA, USA).

\section{Binding ability of targeted nanobubbles in vivo}

Tumor-bearing nude mice were sacrificed at 8 minutes after injection of fluorescence-labeled nanobubbles $\left(5 \times 10^{8} / \mathrm{mL}\right)$, and xenograft tumor tissues were collected. After injection of DiI-labeled targeted nanobubbles, the sections of xenograft tumor tissues were incubated with rat anti-mouse CD31 monoclonal antibody (1:300; Abcam, Cambridge, UK) at $4{ }^{\circ} \mathrm{C}$ overnight and Alexa Fluor ${ }^{\circledR}$ 488-labeled rabbit anti-rat antibody (1:300; Abcam) for 1 hour. After being counterstained with DAPI, the sections were observed under CLSM to evaluate the distribution of targeted nanobubbles. To confirm the ability of targeted nanobubbles to load CAIX aptamer in vivo, the sections of xenograft tumor tissues after injection of 6-FAM and DiI-labeled targeted nanobubbles were stained with DAPI and observed under CLSM. The cryosections of xenograft tumor tissues after injection of DiI-labeled targeted or nontargeted nanobubbles were used to evaluate the binding ability of nanobubbles in xenograft tumor tissues. The sections were incubated with rabbit anti-human CAIX polyclonal antibody (1:200; Abcam) at $4^{\circ} \mathrm{C}$ overnight, then incubated with Alexa Fluor ${ }^{\circledR} 488$-labeled goat antirabbit antibody (1:300; Abcam) for 1 hour, and observed under CLSM.

\section{H\&E staining and CAIX expression}

The sections of xenograft tumor tissues $(\mathrm{n}=9)$ were stained with $\mathrm{H} \& \mathrm{E}$, and the histologic features were described using a DP26 optical microscope (Olympus Optical Co., Ltd., Kyoto, Japan). Xenograft tumor tissues ( $\mathrm{n}=9$ ) were homogenized, and the supernatant was collected. The proteins were separated by $12 \%$ SDS-PAGE and transferred to a polyvinylidene fluoride (PVDF) membrane. The membrane was blocked with 5\% non-fat milk, incubated with rabbit anti-human CAIX monoclonal antibody (1:1,000; Abcam) at $4{ }^{\circ} \mathrm{C}$ overnight and horseradish peroxidase-labeled goat anti-rabbit antibody (1:1,000; Beyotime Institute of Biotechnology) for 1 hour. After incubation with enhanced chemiluminescence reagents, the bands were analyzed using Image $\mathrm{Lab}^{\mathrm{TM}}$ software version 5.2.1 (Bio-Rad Laboratories Inc., Hercules, CA, USA).

\section{Statistical analysis}

Statistical analysis was performed with SPSS 22.0 software (IBM Corporation, Armonk, NY, USA). All quantitative data are presented as the mean \pm SD. Paired-sample $t$-tests and one-way ANOVA were used to compare all parameter indicators. $P<0.05$ was considered statistically significant. GraphPad Prism 6.0 (GraphPad Software, Inc., La Jolla, CA, USA) was used to draw histograms and line graphs. 


\section{Results \\ Specificity of CAIX aptamer}

A single-stranded DNA strand (CAIX aptamer) that bound to CAIX was sequenced by high-throughput sequencing, and the sequence was: AGCAGCACAGAGGTCAGATGT GGTGCGCAGTGATGTGGTTGGTCCTATGCGTGCTACCGTCCTATGCGTGCTACCGTGAA. CLSM showed CAIX aptamer could bind to the cell membranes of 786-O and Hela cells, but could not bind to the cell membranes of BxPC-3 cells, and nonsense aptamer could not bind to the cell membranes of any of the three types of tumor cells (Figure 1A). Flow cytometry found the mean fluorescence intensity of 786-O and Hela cells (37.17 \pm 1.20 au and $34.47 \pm 2.08$ au, respectively) after incubation with CAIX aptamer was significantly stronger than that of 786-O and Hela cells ( $3.66 \pm 0.22$ au and $3.44 \pm 0.10$ au, respectively) after incubation with nonsense aptamer $(P<0.05)$ (Figure 1B and C). However, the mean fluorescence intensity of BxPC-3 cells (3.09 \pm 0.25 au and $3.00 \pm 0.14$ au) was not significantly different after incubation with CAIX aptamer and nonsense aptamer $(P>0.05)$ (Figure 1B and C).
A
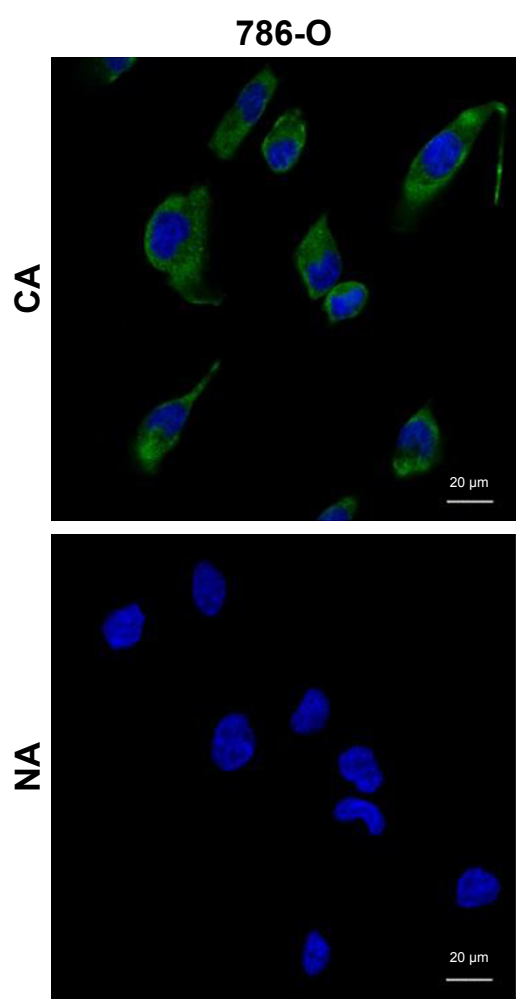

Hela
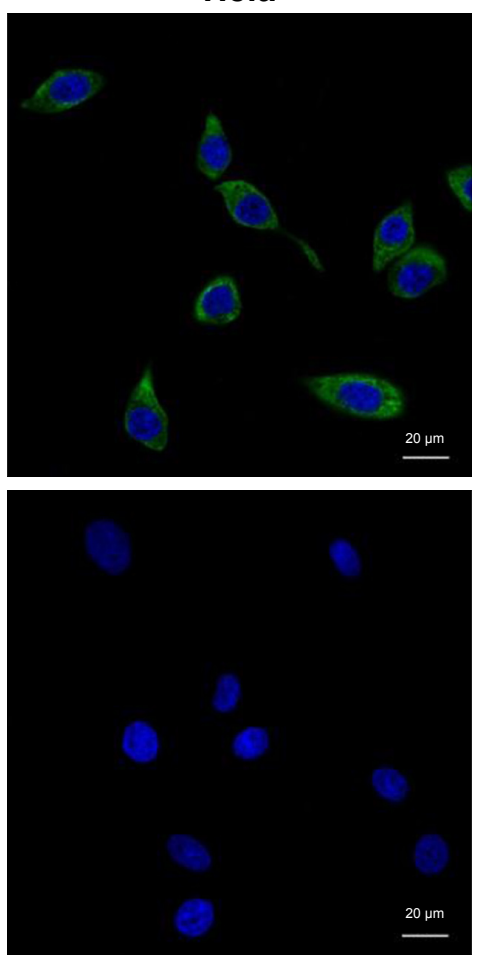

BxPC-3
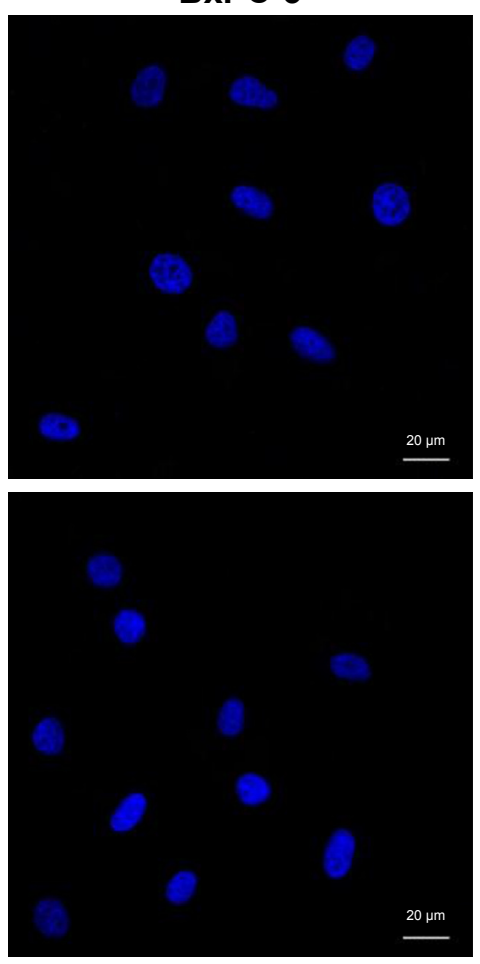

B 786-O

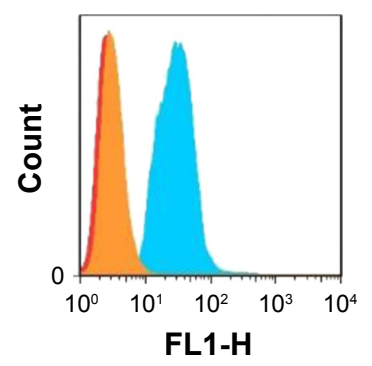

Hela

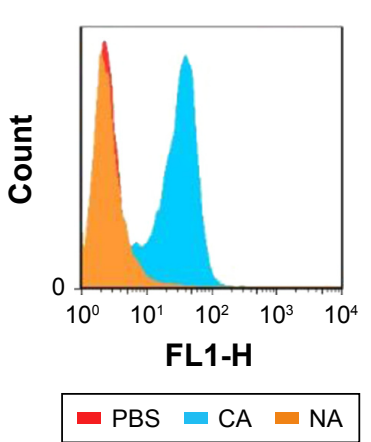

BxPC-3

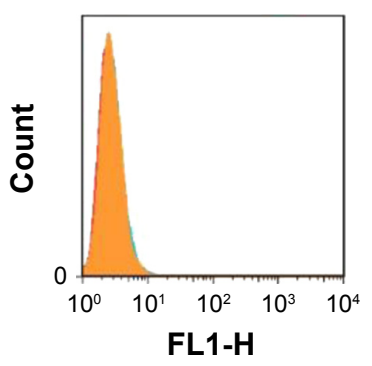

C

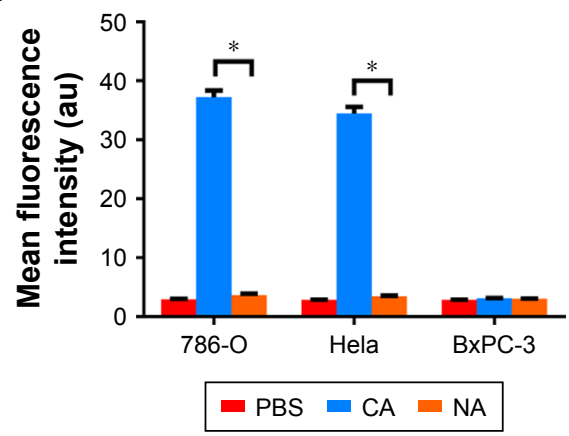

Figure I The specificity of aptamers in cells.

Notes: (A) CLSM images of 786-O cells, Hela cells, and BxPC-3 cells after incubation with CAIX aptamer and nonsense aptamer. (B) Flow cytometry analysis of the binding ability of the two aptamers to 786-O cells, Hela cells, and BxPC-3 cells. (C) Quantification of the binding ability of the two aptamers to the tumor cells $(* P<0.05)$. Abbreviations: CLSM, confocal laser scanning microscopy; CA, CAIX aptamer; CAIX, carbonic anhydrase IX; NA, nonsense aptamer. 


\section{Loading ability of targeted nanobubbles}

The coupling between the surfaces of targeted nanobubbles and CAIX aptamer was the prerequisite for targeted imaging. Under CLSM, the lipid membranes of DiI-labeled targeted nanobubbles presented red fluorescence and 6-FAM-modified CAIX aptamer on targeted nanobubbles presented green fluorescence (Figure 2A and B). The merged image revealed the green fluorescence of 6-FAM-modified CAIX aptamer could overlap with the red fluorescence of the DiI-labeled lipid membranes (Figure 2C). However, nanobubbles unloaded with CAIX aptamer or loaded with no fluorescence-modified CAIX aptamer only presented red fluorescence.

\section{Characteristics of targeted nanobubbles}

A hemocytometer showed the concentration of targeted nanobubbles $\left(3.07 \times 10^{9} \pm 0.29 \times 10^{9} / \mathrm{mL}\right)$ was not significantly different from that of non-targeted nanobubbles loaded with nonsense aptamer $\left(2.99 \times 10^{9} \pm 0.34 \times 10^{9} / \mathrm{mL}\right)(P>0.05)$. The particle size of targeted nanobubbles was $478.43 \pm 68.46 \mathrm{~nm}$, and the polydispersity index was 0.148 (Figure $3 \mathrm{~A}$ ). The particle size of non-targeted nanobubbles was $483.97 \pm 62.08 \mathrm{~nm}$, and the polydispersity index was 0.251 (Figure 3B). Under transmission electron microscope, targeted nanobubbles with uniform distribution and homogeneous size exhibited a black round area encapsulated in a gray ring, whose particle size was about $500 \mathrm{~nm}$ (Figure 3C). The CCK-8 assay demonstrated when the concentration of lipid material reached $15 \mu \mathrm{g} / \mathrm{mL}$, the cell viability of $786-\mathrm{O}$ cells $(87.25 \pm 6.06 \%)$ significantly decreased compared with that of 786-O cells incubated with no lipid material $(P<0.05)$, but when the concentration of lipid material was $10 \mu \mathrm{g} / \mathrm{mL}$, the cell viability of $786-\mathrm{O}$ cells $(91.36 \pm 5.14 \%)$ did not significantly change $(P>0.05)$ (Figure 3D). The imaging effect was not significantly different between targeted and non-targeted nanobubbles at the same concentration in vitro $(P>0.05)$, and the imaging intensity of targeted and non-targeted nanobubbles increased with the increase of concentration (Figure 3E and F).

\section{Binding ability of targeted nanobubbles in vitro}

The binding ability of targeted and non-targeted nanobubbles to the three types of tumor cells was observed under CLSM. A large number of nanobubbles gathered around 786-O and Hela cells after incubation with targeted nanobubbles, while only few nanobubbles could bind to 786-O and Hela cells after incubation with non-targeted nanobubbles, and only few nanobubbles could bind to BxPC-3 cells after incubation with targeted or non-targeted nanobubbles (Figure 4). After the three types of tumor cells were pre-blocked with CAIX aptamer, only a small amount of targeted nanobubbles could bind to the tumor cells (Figure 4).

\section{Imaging effect of targeted nanobubbles in vivo}

Ultrasound images of targeted and non-targeted nanobubbles in 786-O, Hela, and BxPC-3 xenograft tumor tissues are shown in Figure 5A. Qlab 8.1 quantitative analysis software was used to quantitatively analyze the imaging intensity of targeted and non-targeted nanobubbles. The time-intensity curves exhibited that the imaging intensity was always significantly different between targeted and non-targeted nanobubbles in 786-O and Hela xenograft tumor tissues $(P<0.05)$ (Figure 5B and $\mathrm{C}$ ). However, there was no significant difference in the imaging intensity of targeted and non-targeted nanobubbles in BxPC-3 xenograft tumor tissues $(P>0.05)$ (Figure 5D). Peak time of targeted and non-targeted nanobubbles was not significantly different in the three types of xenograft tumor tissues $(P>0.05)$ (Table 1). Peak intensity and area under the curve were significantly different between
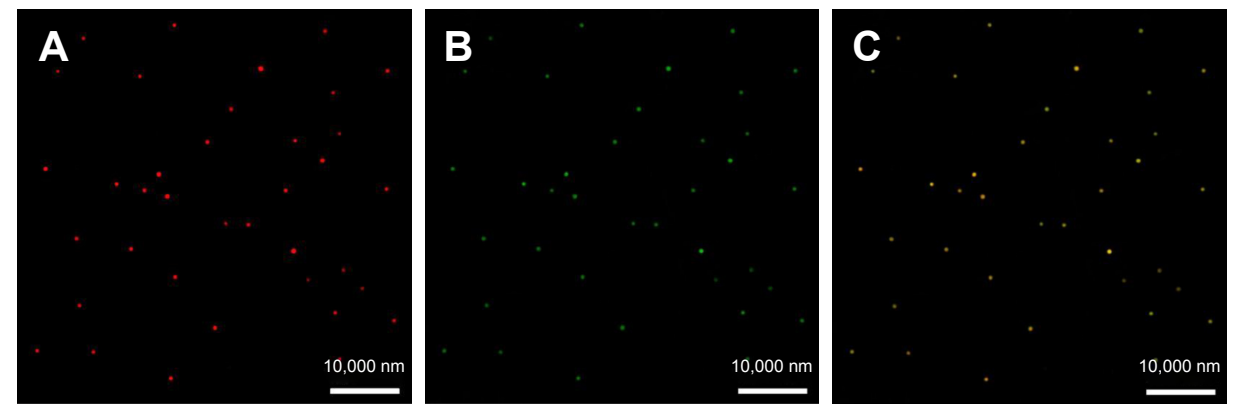

Figure 2 Loading ability of targeted nanobubbles.

Notes: (A) Red fluorescence of Dil-labeled lipid membranes. (B) Green fluorescence of 6-FAM modified CAIX aptamer. (C) The merged image confirms targeted nanobubbles were loaded with CAIX aptamer.

Abbreviations: Dil, I, I'-dioctadecyl-3,3,3',3'-tetramethylindocarbocyanine perchlorate; 6-FAM, 6-carboxyfluorescein; CAIX, carbonic anhydrase IX. 

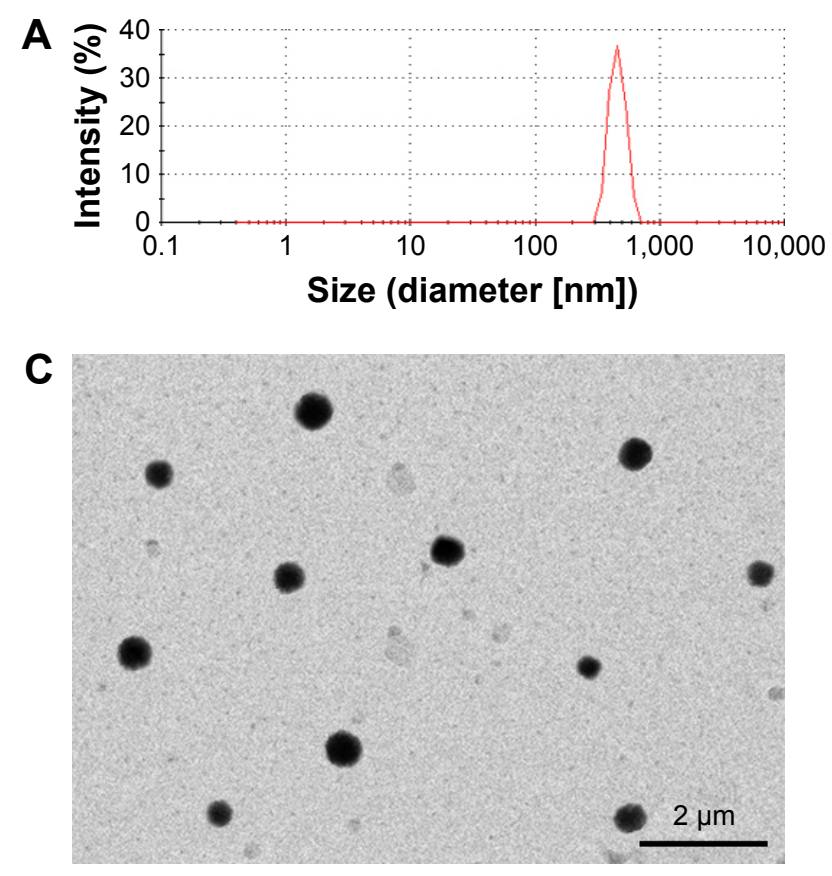

E

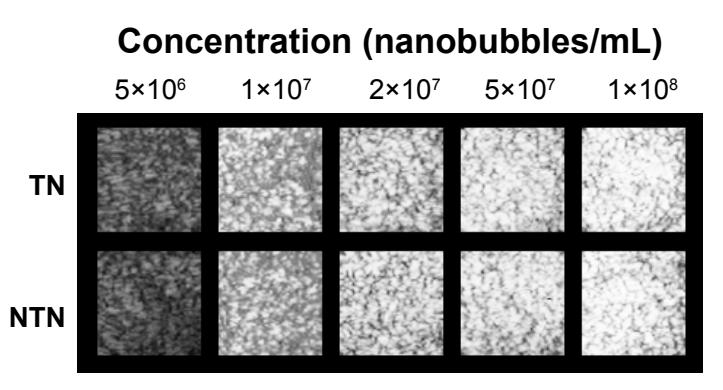

B

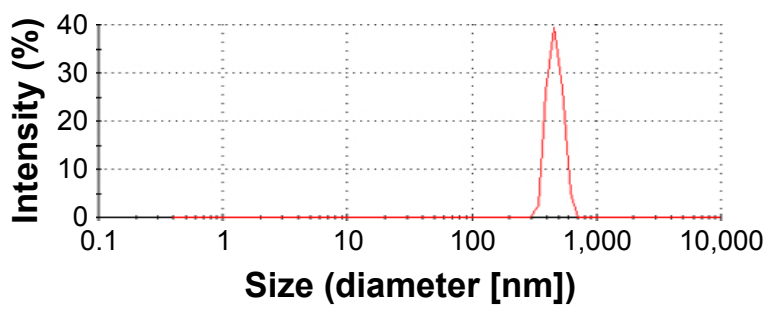

D

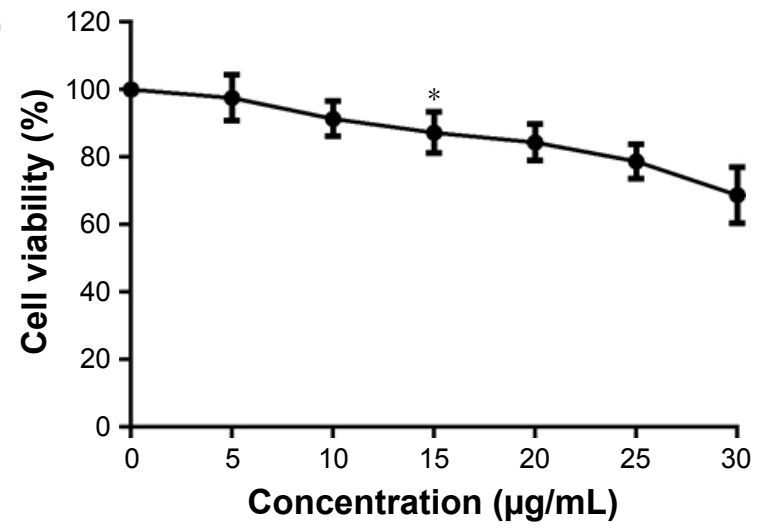

$\mathbf{F}$

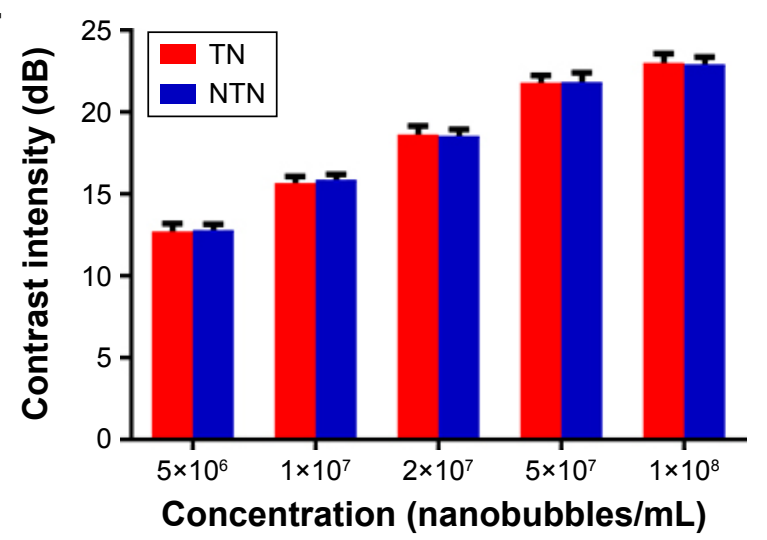

Figure 3 Characteristics of nanobubbles.

Notes: (A) Size-distribution curve of targeted nanobubbles. (B) Size-distribution curve of non-targeted nanobubbles. (C) Observation of targeted nanobubbles under transmission electron microscope. (D) Cell viability of $786-\mathrm{O}$ cells after incubation with lipid material $(* P<0.05$, a significant difference in comparison with the cell viability of 786-O cells incubated with no lipid material). (E) Ultrasound images of nanobubbles in vitro. (F) Quantification of ultrasound images of nanobubbles in vitro.

Abbreviations: TN, targeted nanobubbles; NTN, non-targeted nanobubbles.

targeted and non-targeted nanobubbles in 786-O and Hela xenograft tumor tissues $(P<0.05)$, whereas there was no significant difference in BxPC-3 xenograft tumor tissues $(P>0.05)$ (Table 1).

Fluorescence imaging of live small animals revealed a large number of fluorescent signals were produced in 786-O and Hela xenograft tumor tissues after injection with DiR-labeled targeted nanobubbles, and the fluorescence intensity decreased with time (Figure 6). However, only a few fluorescent signals were presented in BxPC-3 xenograft tumor tissues, and duration time of fluorescent signals was shorter than that in 786-O and Hela xenograft tumor tissues (Figure 6).

\section{Binding ability of targeted nanobubbles in vivo}

The ability of targeted nanobubbles to be distributed in the extravascular spaces and bind to tumor parenchyma cells in xenograft tumor tissues was demonstrated by immunofluorescence. As shown in Figure 7, DiI-labeled targeted nanobubbles were distributed not only in the tumor vasculature, but also in the extravascular spaces of xenograft tumor tissues. As expected, CLSM found the green fluorescence of 6-FAM-modified CAIX aptamer could overlap with the red fluorescence of the lipid membranes of DiI-labeled targeted nanobubbles in xenograft tumor tissues (Figure 8). The specific binding of targeted nanobubbles to tumor parenchymal 

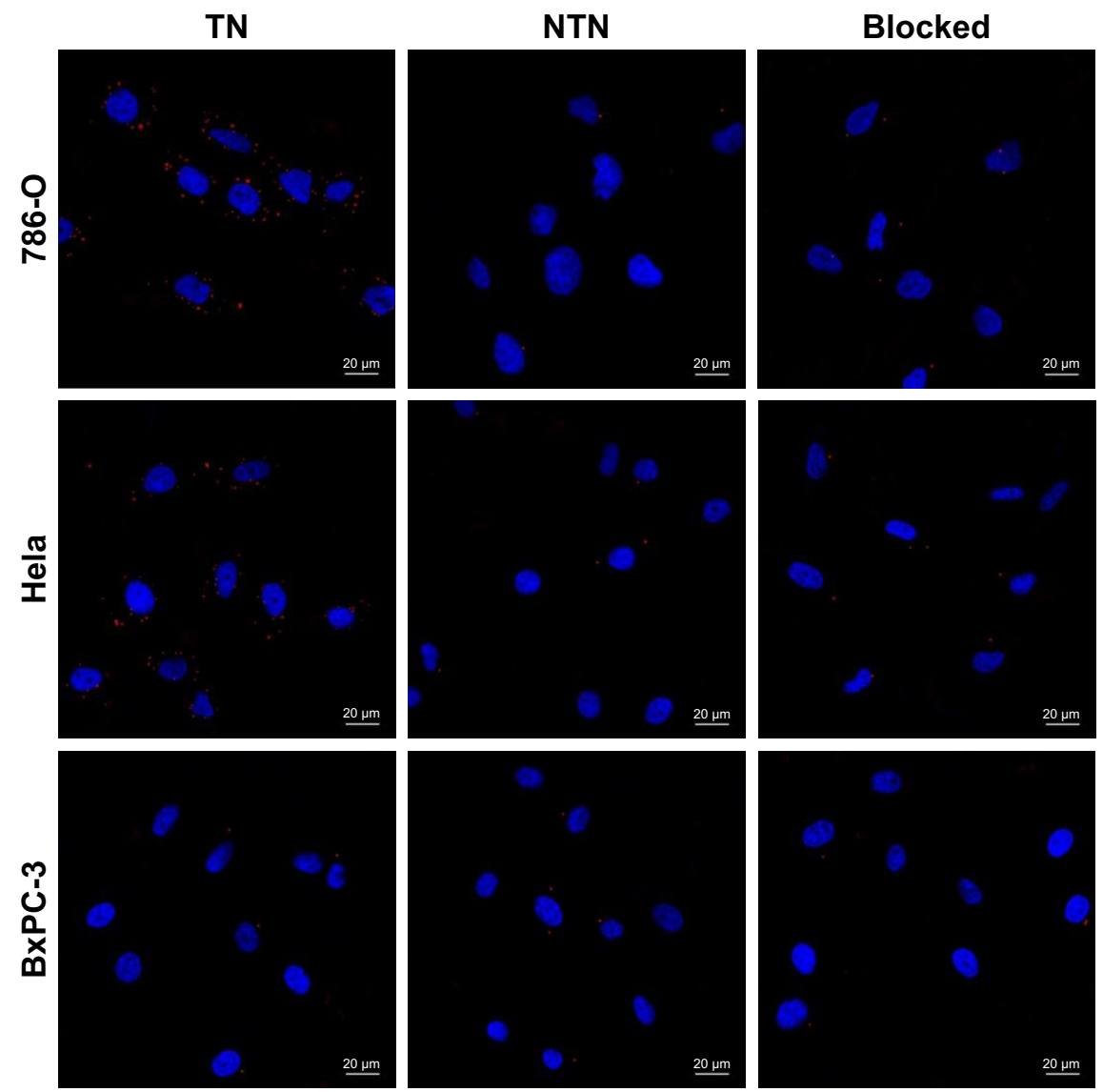

Figure 4 Binding ability of nanobubbles in vitro.

Notes: The binding ability of nanobubbles to 786-O cells (upper), the binding ability of nanobubbles to Hela cells (middle), the binding ability of nanobubbles to BxPC-3 cells (lower). Blocked indicates the tumor cells were pre-blocked with CAIX aptamer. Blue fluorescence indicates cell nuclei, and red fluorescence indicates nanobubbles. Abbreviations: TN, targeted nanobubbles; NTN, non-targeted nanobubbles; CAIX, carbonic anhydrase IX.

cells in xenograft tumor tissues was the key process of USMI. Immunofluorescence described CAIX was expressed in 786-O and Hela xenograft tumor tissues, while not in BxPC-3 xenograft tumor tissues (Figure 9). In 786-O and Hela xenograft tumor tissues, the number was significantly different between targeted and non-targeted nanobubbles $(P<0.05)$, and targeted nanobubbles could gather around CAIX-positive tumor cells (Figure 9A-D). However, the number was not significantly different between targeted and non-targeted nanobubbles in BxPC-3 xenograft tumor tissues $(P>0.05)$ (Figure 9E and $\mathrm{F})$.

\section{H\&E staining and CAIX expression}

H\&E staining showed 786-O xenograft tumor tissues exhibited a cord-like arrangement, and tumor stroma was diffusely distributed in tumor parenchyma (Figure 10A). Tumor cells were obviously heterogeneous, and cell nuclei were large and hyperchromatic (Figure 10A). Hela and BxPC-3 xenograft tumor tissues displayed a sheet or bulk arrangement, and tumor parenchyma was wrapped in tumor stroma (Figure 10B and C). Tumor cells were of different sizes, and cell nuclei were large and had no polarity (Figure 10B and C). Western blot (WB) confirmed the high expression of CAIX in 786-O and Hela xenograft tumor tissues, whereas not in BxPC-3 xenograft tumor tissues (Figure 10D), which was consistent with immunofluorescence.

\section{Discussion}

The early diagnosis of malignant tumors is the key to improving the survival rate and reducing the mortality. ${ }^{32-34}$ Molecular imaging (such as USMI, fluorescent imaging, positron emission tomography, and multimodal imaging) can monitor the biological process in vivo at the cellular and molecular level, which is beneficial to the early diagnosis and treatment evaluation of malignant tumors. ${ }^{35-41}$ The construction of targeted UCAs that can significantly enhance ultrasound imaging is the key link of USMI., ${ }^{7,42,43}$ BR55 is currently the most successful targeted UCAs, which can effectively enhance ultrasound imaging of tumor tissues and has been reported in the early diagnosis of malignant 

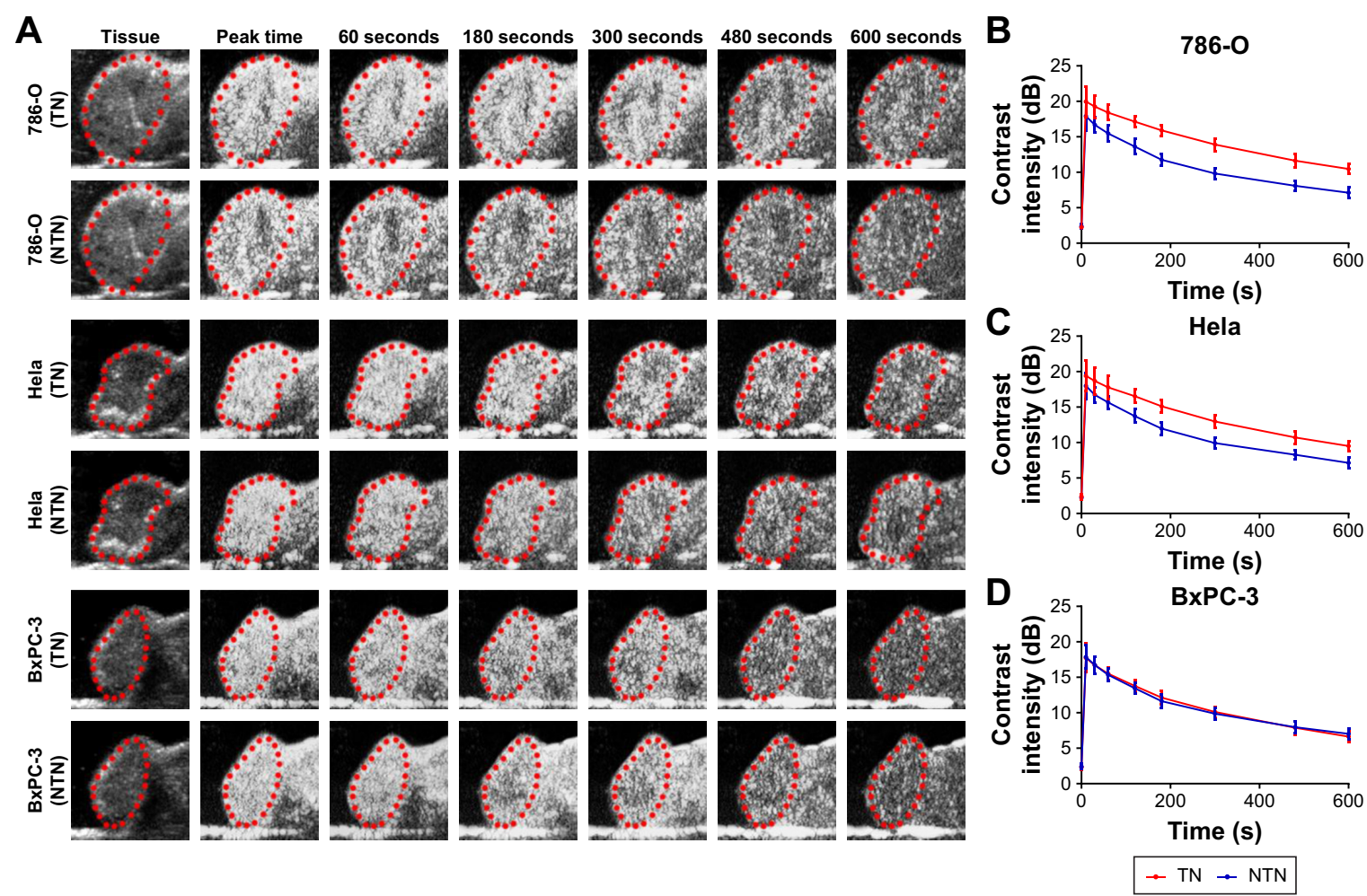

Figure 5 Ultrasound imaging of nanobubbles in vivo.

Notes: (A) Ultrasound images of nanobubbles in xenograft tumor tissues. Red dotted circles indicate the regions of xenograft tumor tissues. (B-D) Time-intensity curves of nanobubbles in 786-O, Hela and BxPC-3 xenograft tumor tissues.

Abbreviations: TN, targeted nanobubbles; NTN, non-targeted nanobubbles.

tumors. ${ }^{44-47}$ However, because the particle size is on the micron scale, BR55 can only achieve intravascular USMI. Nanobubbles with a particle size of less than $700 \mathrm{~nm}$ can penetrate the tumor vasculature to achieve USMI of tumor parenchymal cells and targeted delivery of drugs or genes to tumor parenchymal cells. ${ }^{8,48-51}$

CAIX is a transmembrane protein expressed in numerous tumors, which can be used as a specific target for USMI of malignant tumors. ${ }^{18}$ We have prepared targeted nanobubbles

Table I Ultrasound imaging parameters of targeted and nontargeted nanobubbles in xenografts

\begin{tabular}{lllll}
\hline Tumor & Nanobubbles & $\begin{array}{l}\text { Peak time } \\
\text { (seconds) }\end{array}$ & $\begin{array}{l}\text { Peak } \\
\text { intensity } \\
\text { (dB) }\end{array}$ & AUC (dB·s) \\
\hline $786-O$ & TN & $8.57 \pm 0.34$ & $20.17 \pm 2.32^{*}$ & $8,538.00 \pm 492.01 *$ \\
tumor & NTN & $8.43 \pm 0.58$ & $18.06 \pm 2.11$ & $6,384.50 \pm 485.35$ \\
Hela & TN & $8.47 \pm 0.43$ & $19.68 \pm 2.44^{*}$ & $8,046.63 \pm 521.68 *$ \\
tumor & NTN & $8.55 \pm 0.59$ & $18.18 \pm 1.75$ & $6,461.50 \pm 425.24$ \\
BxPC-3 & TN & $8.53 \pm 0.78$ & $17.86 \pm 2.07$ & $6,410.88 \pm 477.79$ \\
tumor & NTN & $8.61 \pm 0.73$ & $18.02 \pm 1.72$ & $6,340.38 \pm 482.47$ \\
\hline
\end{tabular}

Note: $*$ A significant difference $(P<0.05)$ in comparison with the same ultrasound imaging parameter of non-targeted nanobubbles.

Abbreviations: AUC, area under the curve; TN, targeted nanobubbles; NTN, nontargeted nanobubbles. loaded with CAIX polypeptides in our previous research, which could be used for USMI of CAIX-positive xenograft tumor tissues. ${ }^{23}$ However, polypeptides are composed of amino acid sequences, which can cause an immune response after repeated injection, thus restricting their clinical application. ${ }^{52}$ Aptamers are composed of nucleic acid sequences and have been widely applied in molecular diagnosis and disease treatment, including USMI of malignant tumors. ${ }^{15,50}$

Therefore, this study combined lipid nanobubbles with small particle size, CAIX expressed in numerous tumors, and safe and stable aptamers to construct targeted nanobubbles loaded with CAIX aptamer. CAIX aptamer could specifically bind to CAIX-positive 786-O and Hela cells, rather than CAIX-negative BxPC-3 cells, which laid the foundation for the specific binding of targeted nanobubbles loaded with CAIX aptamer to CAIX-positive tumor cells. Compared with the traditional biotin-avidin system, the maleimide-thiol coupling reaction not only loaded specific ligands on targeted nanobubbles, but also did not cause an immune response after intravenous injection of targeted nanobubbles. ${ }^{31,53}$ Therefore, targeted nanobubbles loaded with CAIX aptamer were prepared by a maleimide-thiol coupling reaction in this 


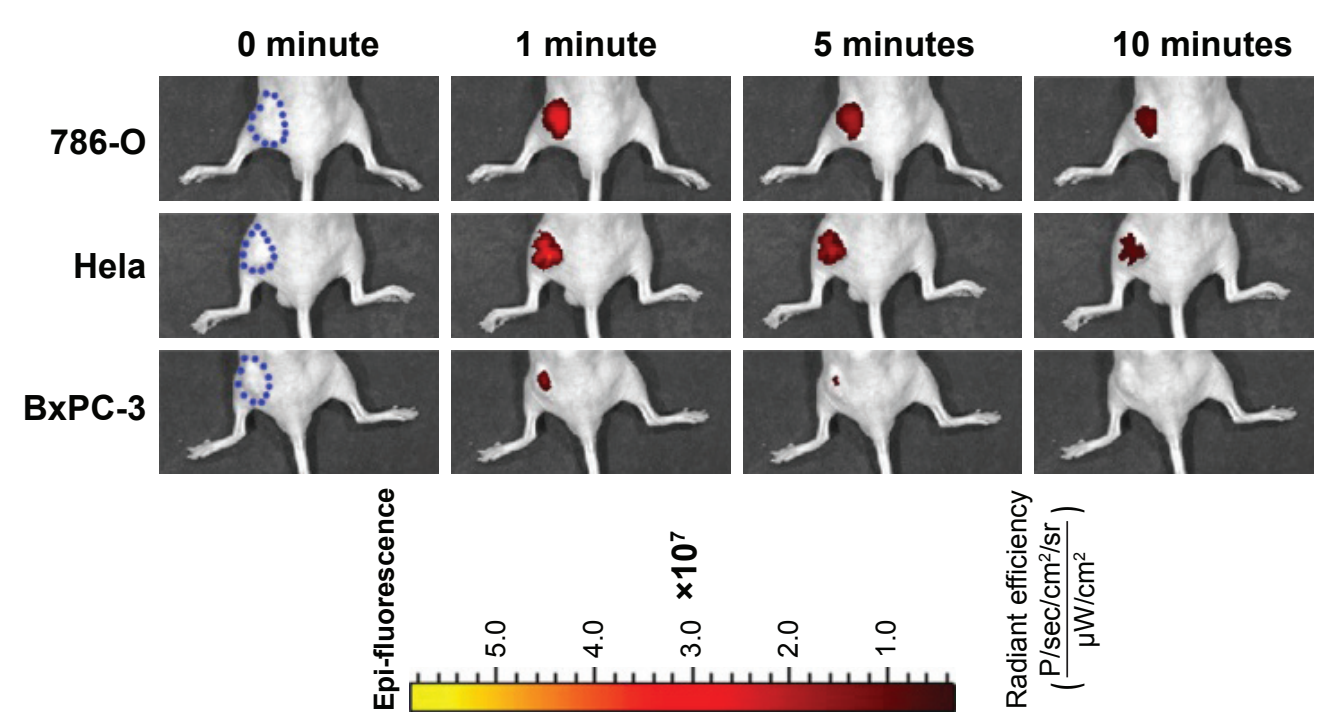

Figure 6 Fluorescence imaging of xenograft tumor tissues of live small animals.

Notes: Fluorescence imaging of live small animals was performed in 786-O, Hela, and BxPC-3 tumor-bearing nude mice over time $(0$ minute, 1 minute, 5 minutes, and 10 minutes). Blue dotted circles indicate the regions of xenograft tumor tissues.
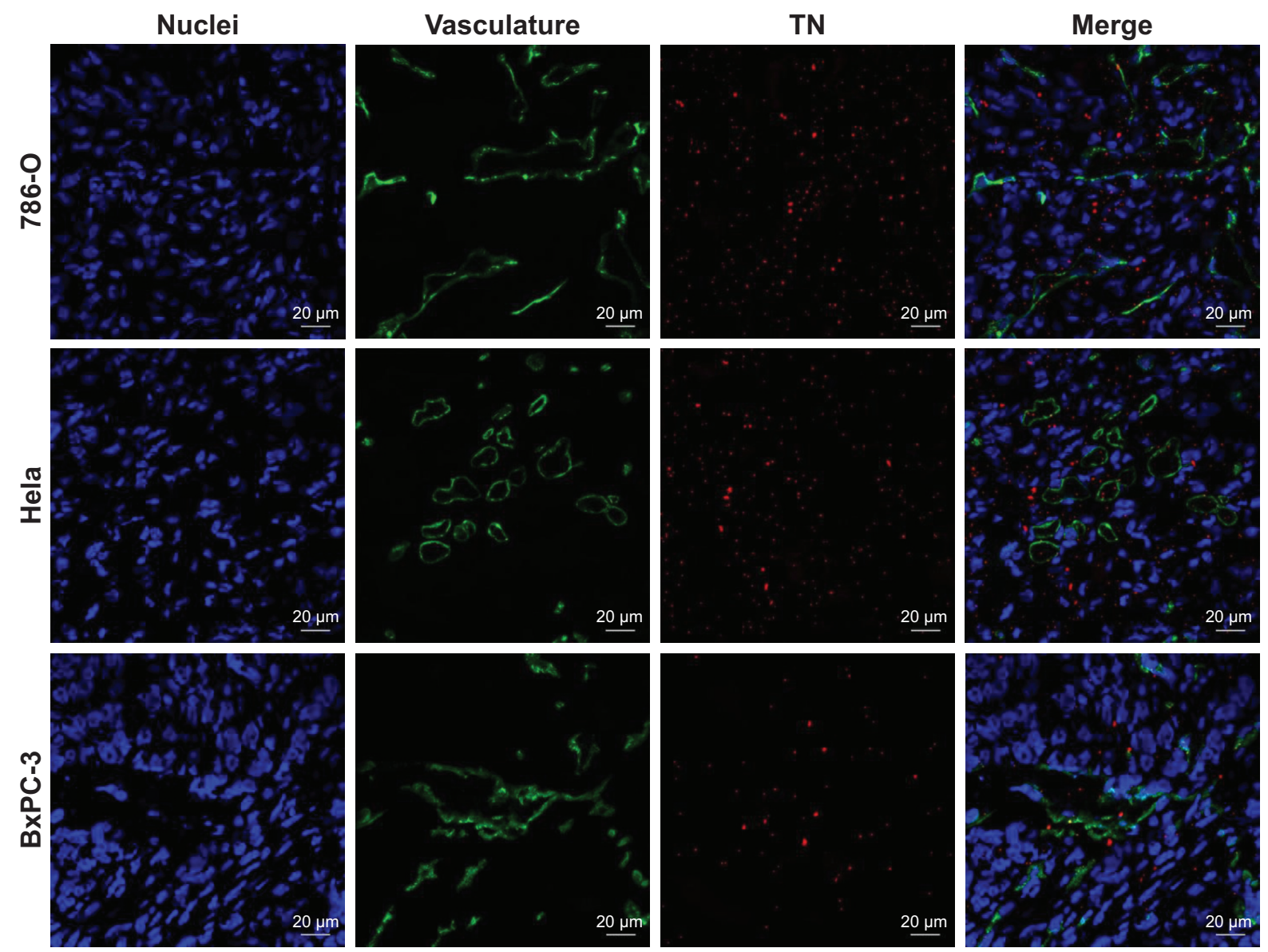

Figure 7 Distribution of targeted nanobubbles in xenograft tumor tissues.

Notes: The distribution of targeted nanobubbles in 786-O xenograft tumor tissues (upper), in Hela xenograft tumor tissues (middle), and in BxPC-3 xenograft tumor tissues (lower). Blue fluorescence indicates cell nuclei, green fluorescence indicates tumor vasculature, and red fluorescence indicates nanobubbles.

Abbreviation: TN, targeted nanobubbles. 

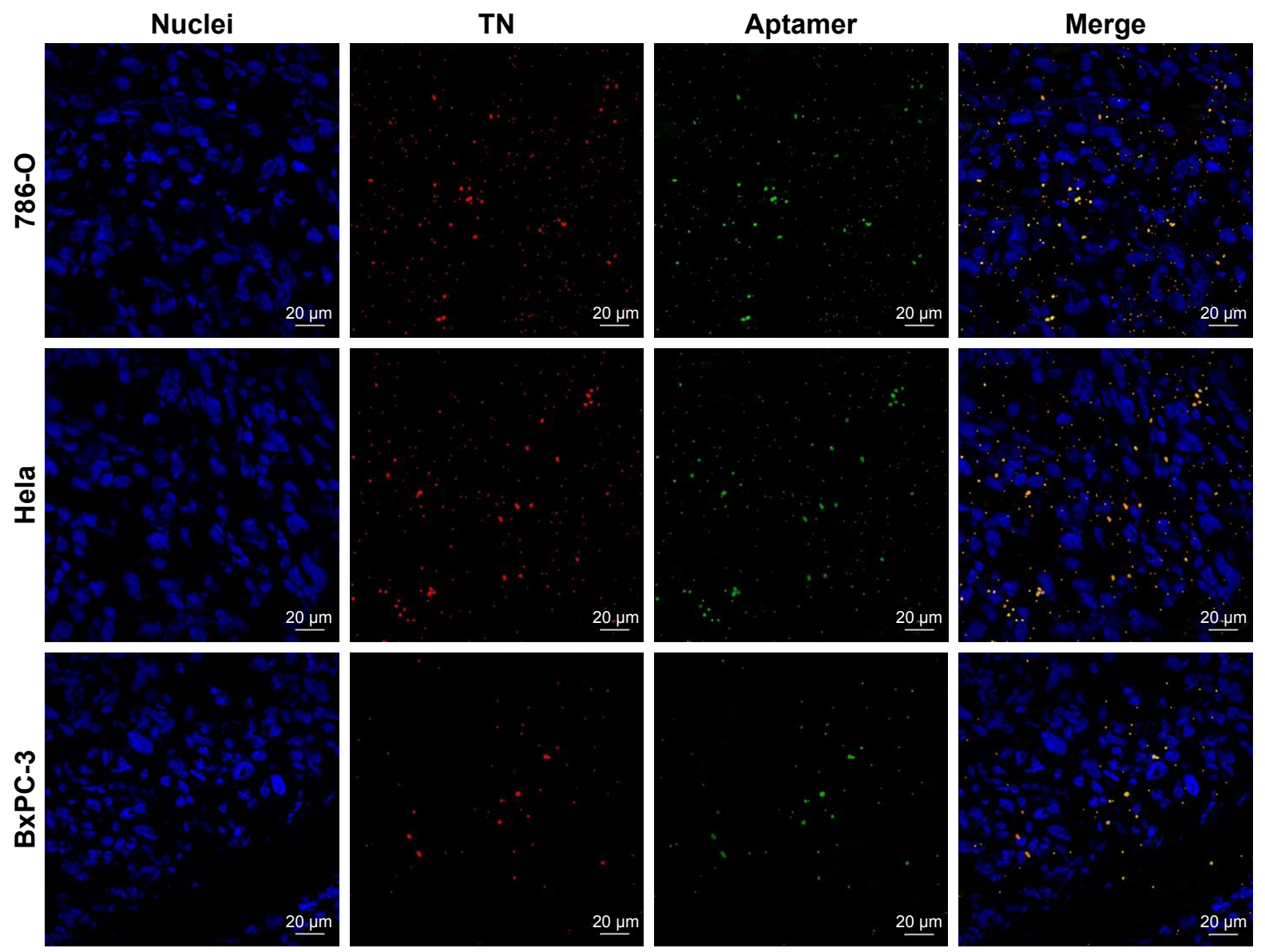

Figure 8 Ability of targeted nanobubbles to load CAIX aptamer in xenograft tumor tissues.

Notes: The ability of targeted nanobubbles to load CAIX aptamer in 786-O xenograft tumor tissues (upper), in Hela xenograft tumor tissues (middle), and in BxPC-3 xenograft tumor tissues (lower). Blue fluorescence indicates cell nuclei, red fluorescence indicates the lipid membranes of targeted nanobubbles, and green fluorescence indicates CAIX aptamer.

Abbreviations: TN, targeted nanobubbles; CAIX, carbonic anhydrase IX.

study, and fluorescence labeling confirmed CAIX aptamer was distributed uniformly on targeted nanobubbles. Targeted nanobubbles were round and distributed uniformly, and their particle size was $478.43 \pm 68.46 \mathrm{~nm}$. In in vitro experiments, targeted nanobubbles could specifically bind to 786-O and Hela cells, and the binding ability of targeted nanobubbles to CAIX-positive tumor cells could be blocked by CAIX aptamer. It was considered that targeted nanobubbles were specific to CAIX-positive tumor cells.

The essence of USMI is to specifically enhance ultrasound imaging of tumor tissues with UCAs. Targeted nanobubbles had a better imaging effect in 786-O and Hela xenograft tumor tissues compared with non-targeted nanobubbles loaded with nonsense aptamer, which displayed higher peak intensity and larger area under the curve. However, there was no significant difference in the imaging effects of targeted and non-targeted nanobubbles in BxPC-3 xenograft tumor tissues.
These attributes suggested targeted nanobubbles loaded with CAIX aptamer could specifically gather in CAIX-positive xenograft tumor tissues compared with non-targeted nanobubbles, whereas the aggregation ability of targeted and non-targeted nanobubbles was not significantly different in CAIX-negative xenograft tumor tissues. Fluorescence imaging of live small animals showed only a few fluorescent signals were seen in BxPC-3 xenograft tumor tissues, and duration time was short. The reason was that only a few targeted nanobubbles gathered in CAIX-negative xenograft tumor tissues, and DiR dye on targeted nanobubbles was not enough for fluorescence imaging in BxPC-3 xenograft tumor tissues over time.

The morphological basis of USMI of tumor parenchyma cells includes targeted nanobubbles penetrating the tumor vasculature and binding to tumor cells by specific ligands on their surfaces. Because the imaging intensity of 

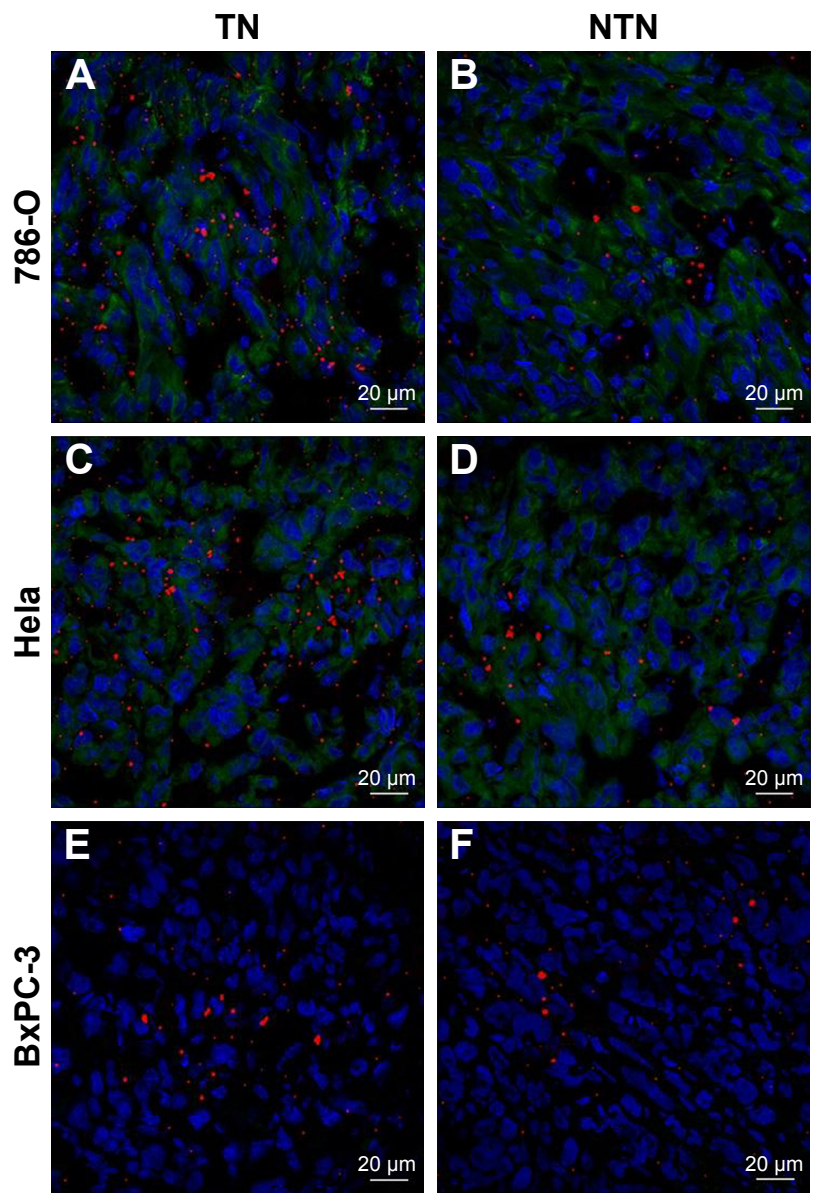

Figure 9 Binding ability of nanobubbles in xenograft tumor tissues.

Notes: (A, B) The binding ability of nanobubbles to tumor cells in 786-O xenograft tumor tissues, (C, D) in Hela xenograft tumor tissues, and (E, F) in BxPC-3 xenograft tumor tissues. Blue fluorescence indicates cell nuclei, green fluorescence indicates the cell membranes of CAIX-positive cells, and red fluorescence indicates nanobubbles.

Abbreviations: TN, targeted nanobubbles; NTN, non-targeted nanobubbles; CAIX, carbonic anhydrase IX.

tumor tissues mainly came from bound targeted UCAs at 8 minutes after injection of targeted UCAs, we observed the sections at 8 minutes after injection of nanobubbles to investigate the morphological basis of USMI.,52 Targeted nanobubbles were seen in the vascular and extravascular spaces of 786-O, Hela, and BxPC-3 xenograft tumor tissues, which meant targeted nanobubbles could penetrate the tumor vasculature of xenograft tumor tissues. Fluorescence labeling found targeted nanobubbles distributed in the extravascular spaces of xenograft tumor tissues could still load CAIX aptamer. In addition, immunofluorescence not only displayed the distribution differences of targeted and non-targeted nanobubbles in 786-O and Hela xenograft tumor tissues, but also demonstrated targeted nanobubbles could specifically bind to CAIX-positive tumor cells in 786-O and Hela xenograft tumor tissues, whereas not to
CAIX-negative tumor cells in BxPC-3 xenograft tumor tissues. WB further showed the high expression of CAIX in 786-O and Hela xenograft tumor tissues, while not in BxPC-3 xenograft tumor tissues. Therefore, the morphological basis of USMI of malignant tumors after intravenous injection of targeted nanobubbles had been illustrated, namely, targeted nanobubbles in circulation could passively diffuse into the extravascular spaces via EPR effect, actively bind to CAIX-positive tumor cells under the action of CAIX aptamer, significantly enhance ultrasound imaging of CAIX-positive xenograft tumor tissues, and thus achieve USMI of tumor parenchyma cells.

This study had the following characteristics: 1) aptamermediated targeted nanobubbles were constructed, which could specifically bind to tumor cells and safely and effectively enhance ultrasound imaging of tumor tissues; 2) targeted nanobubbles were constructed by a maleimide-thiol coupling reaction, which could not only load CAIX aptamer on targeted nanobubbles stably and effectively, but also avoid an immune response in vivo; 3 ) because numerous tumors express CAIX, targeted nanobubbles loaded with CAIX aptamer can be used for USMI of various tumors; 4) the distribution, loading ability, and binding ability of targeted nanobubbles in xenograft tumor tissues were investigated in depth, which fully clarified the morphological basis of USMI. Though targeted nanobubbles could load CAIX aptamer and specifically bind to CAIX-positive tumor cells in vitro and in vivo, future experiments need to investigate the relationship between the number of CAIX aptamer molecules on targeted nanobubbles and the binding ability of targeted nanobubbles to CAIX.

\section{Conclusion}

Combining the advantages of nanobubbles and aptamers, this study successfully constructed targeted nanobubbles loaded with CAIX aptamer by a maleimide-thiol coupling reaction, which possessed the attractive features of small size, strong penetration, high safety, high specificity, and excellent imaging effect. Furthermore, the distribution of targeted nanobubbles in the extravascular spaces and the ability to load CAIX aptamer and bind to CAIX-positive tumor cells in xenograft tumor tissues were confirmed by immunofluorescence. This study not only introduces targeted nanobubbles carrying aptamers for USMI of malignant tumors from various organs, but also further determines the morphological basis of USMI of malignant tumors after intravenous injection of targeted nanobubbles and lays the foundation for the application of targeted nanobubbles in the early diagnosis of malignant tumors. 

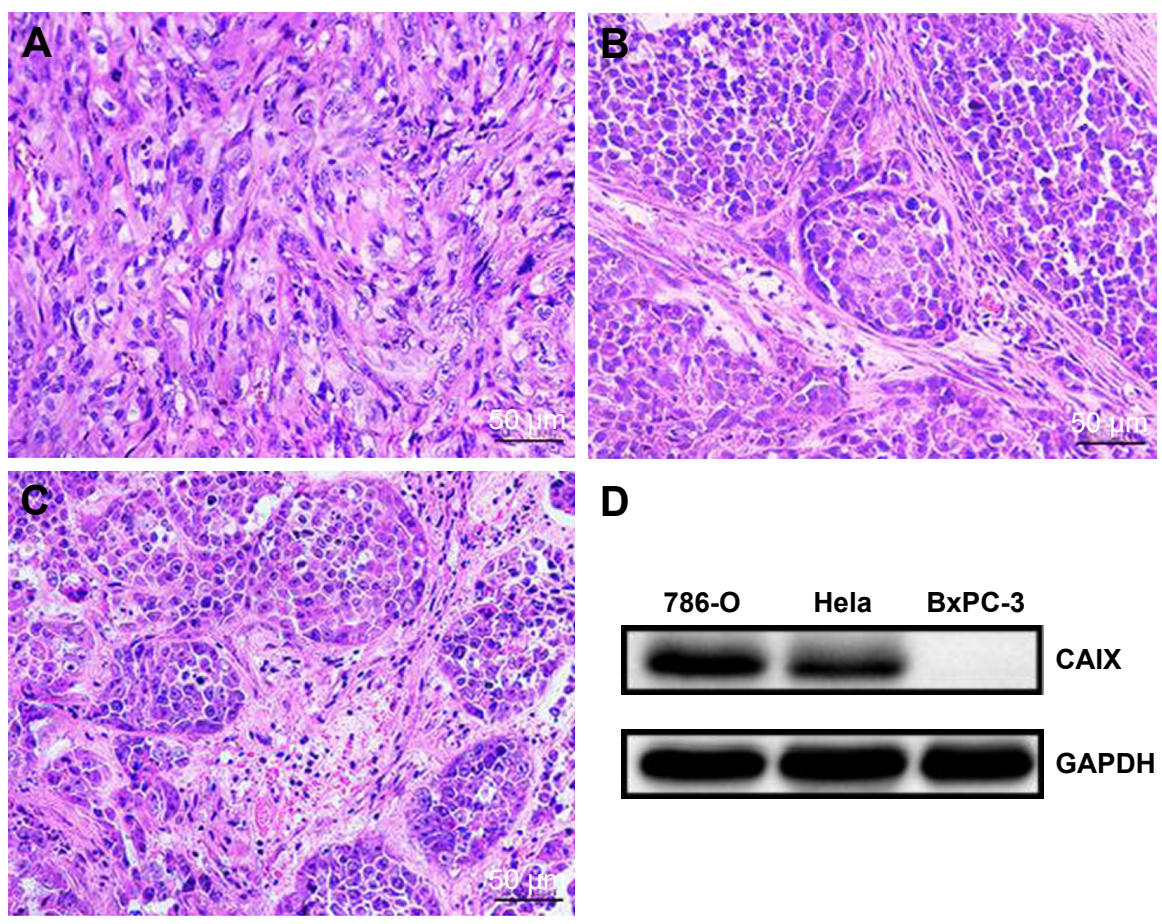

D

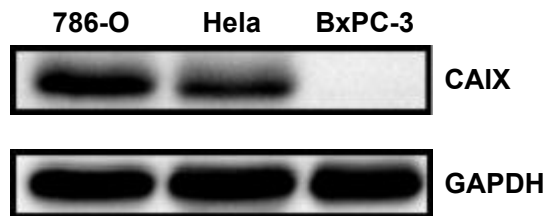

Figure $10 \mathrm{H} \& \mathrm{E}$ staining and CAIX expression of xenograft tumor tissues.

Notes: (A) 786-O xenograft tumor tissues stained with H\&E. (B) Hela xenograft tumor tissues stained with H\&E. (C) BxPC-3 xenograft tumor tissues stained with H\&E. (D) Expression of CAIX in xenograft tumor tissues.

Abbreviations: CAIX, carbonic anhydrase IX; GAPDH, glyceraldehyde-3-phosphate dehydrogenase.

\section{Acknowledgment}

This work was supported by the International Science \& Technology Cooperation Program of China (No 2015DFA30920), the National Natural Science Foundation of China (No 81771856), and the Science \& Technology (International Science \& Technology Cooperation) Research Base Construction Program of Chongqing (No cstc2014gjhz110004).

\section{Disclosure}

The authors report no conflicts of interest in this work.

\section{References}

1. Terzi E, Iavarone M, Pompili M, et al. Contrast ultrasound LI-RADS LR-5 identifies hepatocellular carcinoma in cirrhosis in a multicenter restropective study of 1,006 nodules. J Hepatol. 2018;68(3):485-492.

2. Abou-Elkacem L, Wilson KE, Johnson SM, et al. Ultrasound Molecular Imaging of the Breast Cancer Neovasculature using Engineered Fibronectin Scaffold Ligands: A Novel Class of Targeted Contrast Ultrasound Agent. Theranostics. 2016;6(11):1740-1752.

3. Bzyl J, Palmowski M, Rix A, et al. The high angiogenic activity in very early breast cancer enables reliable imaging with VEGFR2-targeted microbubbles (BR55). Eur Radiol. 2013;23(2):468-475.

4. Zhou Y, Gu H, Xu Y, et al. Targeted antiangiogenesis gene therapy using targeted cationic microbubbles conjugated with CD105 antibody compared with untargeted cationic and neutral microbubbles. Theranostics. 2015;5(4):399-417.

5. Zhang H, Ingham ES, Gagnon MK, et al. Invitro characterization and invivo ultrasound molecular imaging of nucleolin-targeted microbubbles. Biomaterials. 2017;118:63-73.
6. Bachawal SV, Jensen KC, Wilson KE, Tian L, Lutz AM, Willmann JK Breast Cancer Detection by B7-H3-Targeted Ultrasound Molecular Imaging. Cancer Res. 2015;75(12):2501-2509.

7. Cai WB, Yang HL, Zhang J, et al. The Optimized Fabrication of Nanobubbles as Ultrasound Contrast Agents for Tumor Imaging. Sci Rep. 2015;5:13725.

8. Li J, Tian Y, Shan D, et al. Neuropeptide $Y Y_{1}$ receptor-mediated biodegradable photoluminescent nanobubbles as ultrasound contrast agents for targeted breast cancer imaging. Biomaterials. 2017;116: 106-117.

9. Yang H, Cai W, Xu L, et al. Nanobubble-Affibody: Novel ultrasound contrast agents for targeted molecular ultrasound imaging of tumor. Biomaterials. 2015;37:279-288.

10. Fan X, Wang L, Guo Y, Xiong X, Zhu L, Fang K. Inhibition of prostate cancer growth using doxorubicin assisted by ultrasound-targeted nanobubble destruction. Int J Nanomedicine. 2016;11:3585-3596.

11. Segers T, de Rond L, de Jong N, Borden M, Versluis M. Stability of Monodisperse Phospholipid-Coated Microbubbles Formed by Flow-Focusing at High Production Rates. Langmuir. 2016;32(16): 3937-3944.

12. Perera $\mathrm{RH}, \mathrm{Wu} \mathrm{H}$, Peiris $\mathrm{P}$, et al. Improving performance of nanoscale ultrasound contrast agents using N,N-diethylacrylamide stabilization. Nanomedicine. 2017;13(1):59-67.

13. Duan S, Guo L, Shi D, Shang M, Meng D, Li J. Development of a novel folate-modified nanobubbles with improved targeting ability to tumor cells. Ultrason Sonochem. 2017;37:235-243.

14. Gao Y, Hernandez C, Yuan HX, et al. Ultrasound molecular imaging of ovarian cancer with CA-125 targeted nanobubble contrast agents. Nanomedicine. 2017;13(7):2159-2168.

15. Fan X, Guo Y, Wang L, Xiong X, Zhu L, Fang K. Diagnosis of prostate cancer using anti-PSMA aptamer A10-3.2-oriented lipid nanobubbles. Int J Nanomedicine. 2016;11:3939-3950.

16. Chang SS. Overview of prostate-specific membrane antigen. Rev Urol. 2004;6 (Suppl 10):S13-S18. 
17. Tang L, Tong R, Coyle VJ, et al. Targeting tumor vasculature with aptamer-functionalized doxorubicin-polylactide nanoconjugates for enhanced cancer therapy. ACS Nano. 2015;9(5):5072-5081.

18. Garousi J, Honarvar H, Andersson KG, et al. Comparative Evaluation of Affibody Molecules for Radionuclide Imaging of in Vivo Expression of Carbonic Anhydrase IX. Mol Pharm. 2016;13(11):3676-3687.

19. Ellingsen C, Andersen LM, Galappathi K, Rofstad EK. Hypoxia biomarkers in squamous cell carcinoma of the uterine cervix. BMC Cancer. 2015;15:805.

20. Coviello V, Marchi B, Sartini S, et al. 1,2-Benzisothiazole Derivatives Bearing 4-, 5-, or 6-Alkyl/arylcarboxamide Moieties Inhibit Carbonic Anhydrase Isoform IX (CAIX) and Cell Proliferation under Hypoxic Conditions. J Med Chem. 2016;59(13):6547-6552.

21. Viola-Villegas NT, Carlin SD, Ackerstaff E, et al. Understanding the pharmacological properties of a metabolic PET tracer in prostate cancer. Proc Natl Acad Sci US A. 2014;111(20):7254-7259.

22. van Brussel AS, Adams A, Oliveira S, et al. Hypoxia-Targeting Fluorescent Nanobodies for Optical Molecular Imaging of Pre-Invasive Breast Cancer. Mol Imaging Biol. 2016;18(4):535-544.

23. Zegers CM, Hoebers FJ, van Elmpt W, et al. Evaluation of tumour hypoxia during radiotherapy using $\left[{ }^{18} \mathrm{~F}\right] \mathrm{HX} 4 \mathrm{PET}$ imaging and blood biomarkers in patients with head and neck cancer. Eur J Nucl Med Mol Imaging. 2016;43(12):2139-2146.

24. Muselaers CH, Rijpkema M, Bos DL, et al. Radionuclide and Fluorescence Imaging of Clear Cell Renal Cell Carcinoma Using Dual Labeled Anti-Carbonic Anhydrase IX Antibody G250. J Urol. 2015; 194(2):532-538.

25. Zhu L, Guo Y, Wang L, et al. Construction of ultrasonic nanobubbles carrying CAIX polypeptides to target carcinoma cells derived from various organs. J Nanobiotechnology. 2017;15(1):63.

26. Wu Q, Wu L, Wang Y, et al. Evolution of DNA aptamers for malignant brain tumor gliosarcoma cell recognition and clinical tissue imaging. Biosens Bioelectron. 2016;80:1-8.

27. Kang SA, Tsolmon B, Mann AP, et al. Safety evaluation of intravenously administered mono-thioated aptamer against E-selectin in mice. Toxicol Appl Pharmacol. 2015;287(1):86-92.

28. Song Y, Zhu Z, An Y, et al. Selection of DNA aptamers against epithelial cell adhesion molecule for cancer cell imaging and circulating tumor cell capture. Anal Chem. 2013;85(8):4141-4149.

29. Alibolandi M, Ramezani M, Abnous $\mathrm{K}$, et al. In vitro and in vivo evaluation of therapy targeting epithelial-cell adhesion-molecule aptamers for non-small cell lung cancer. J Control Release. 2015;209: $88-100$.

30. Jacobson O, Weiss ID, Wang L, et al. 18F-Labeled Single-Stranded DNA Aptamer for PET Imaging of Protein Tyrosine Kinase-7 Expression. J Nucl Med. 2015;56(11):1780-1785.

31. Gao Z, Hou Y, Zeng J, et al. Tumor Microenvironment-Triggered Aggregation of Antiphagocytosis ${ }^{99 \mathrm{~m}}$ Tc-Labeled $\mathrm{Fe}_{3} \mathrm{O}_{4}$ Nanoprobes for Enhanced Tumor Imaging In Vivo. Adv Mater. 2017;29(24): 1701095.

32. Chen W, Zheng R, Baade PD, et al. Cancer statistics in China, 2015. CA Cancer J Clin. 2016;66(2):115-132.

33. Chen $\mathrm{W}$, Zheng R, Zhang S, et al. Cancer incidence and mortality in China, 2013. Cancer Lett. 2017;401:63-71.

34. Doroudi M, Schoen RE, Pinsky PF. Early detection versus primary prevention in the PLCO flexible sigmoidoscopy screening trial: Which has the greatest impact on mortality? Cancer. 2017;123(24): 4815-4822.

35. Liu J, Chen Y, Wang G, et al. Ultrasound molecular imaging of acute cardiac transplantation rejection using nanobubbles targeted to T lymphocytes. Biomaterials. 2018;162:200-207.
36. Zhou T, Cai W, Yang H, et al. Annexin V conjugated nanobubbles: A novel ultrasound contrast agent for in vivo assessment of the apoptotic response in cancer therapy. J Control Release. 2018;276:113-124.

37. Wang S, Herbst EB, Mauldin FW, Diakova GB, Klibanov AL, Hossack JA. Ultra-Low-Dose Ultrasound Molecular Imaging for the Detection of Angiogenesis in a Mouse Murine Tumor Model: How Little Can We See? Invest Radiol. 2016;51(12):758-766.

38. Sun Y, Ma X, Cheng K, et al. Strained cyclooctyne as a molecular platform for construction of multimodal imaging probes. Angew Chem Int Ed Engl. 2015;54(20):5981-5984.

39. Ding F, Chen S, Zhang W, Tu Y, Sun Y. UPAR targeted molecular imaging of cancers with small molecule-based probes. Bioorg Med Chem. 2017;25(20):5179-5184.

40. Ding F, Zhan Y, Lu X, Sun Y. Recent advances in near-infrared II fluorophores for multifunctional biomedical imaging. Chem Sci. 2018;9(19):4370-4380.

41. Sun Y, Zeng X, Xiao Y, et al. Novel dual-function near-infrared II fluorescence and PET probe for tumor delineation and image-guided surgery. Chem Sci. 2018;9(8):2092-2097.

42. Lutz AM, Bachawal SV, Drescher CW, Pysz MA, Willmann JK, Gambhir SS. Ultrasound molecular imaging in a human CD276 expression-modulated murine ovarian cancer model. Clin Cancer Res. 2014;20(5):1313-1322.

43. Hoyt K, Warram JM, Wang D, Ratnayaka S, Traylor A, Agarwal A. Molecular Ultrasound Imaging of Tissue Inflammation Using an Animal Model of Acute Kidney Injury. Mol Imaging Biol. 2015;17(6):786-792.

44. Baron Toaldo M, Salvatore V, Marinelli S, et al. Use of VEGFR-2 targeted ultrasound contrast agent for the early evaluation of response to sorafenib in a mouse model of hepatocellular carcinoma. Mol Imaging Biol. 2015;17(1):29-37.

45. Hackl C, Schacherer D, Anders M, et al. Improved Detection of preclinical Colorectal Liver Metastases by High Resolution Ultrasound including Molecular Ultrasound Imaging using the targeted Contrast Agent BR55. Ultraschall Med. 2016;37(3):290-296.

46. Willmann JK, Bonomo L, Carla Testa A, et al. Ultrasound Molecular Imaging With BR55 in Patients With Breast and Ovarian Lesions: First-in-Human Results. J Clin Oncol. 2017;35(19):2133-2140.

47. Smeenge M, Tranquart F, Mannaerts CK, et al. First-in-Human Ultrasound Molecular Imaging With a VEGFR2-Specific Ultrasound Molecular Contrast Agent (BR55) in Prostate Cancer: A Safety and Feasibility Pilot Study. Invest Radiol. 2017;52(7):419-427.

48. Oda Y, Suzuki R, Mori T, et al. Development of fluorous lipid-based nanobubbles for efficiently containing perfluoropropane. Int J Pharm. 2015;487(1-2):64-71.

49. Ho YJ, Chang YC, Yeh CK. Improving Nanoparticle Penetration in Tumors by Vascular Disruption with Acoustic Droplet Vaporization. Theranostics. 2016;6(3):392-403.

50. Wu M, Wang Y, Wang Y, et al. Paclitaxel-loaded and A10-3.2 aptamertargeted poly(lactide-co-glycolic acid) nanobubbles for ultrasound imaging and therapy of prostate cancer. Int J Nanomedicine. 2017; 12:5313-5330.

51. Wu M, Zhao H, Guo L, et al. Ultrasound-mediated nanobubble destruction (UMND) facilitates the delivery of A10-3.2 aptamer targeted and siRNA-loaded cationic nanobubbles for therapy of prostate cancer. Drug Deliv. 2018;25(1):226-240.

52. Zhang H, Tam S, Ingham ES, et al. Ultrasound molecular imaging of tumor angiogenesis with a neuropilin-1-targeted microbubble. Biomaterials. 2015;56:104-113.

53. Hou Y, Qiao R, Fang F, et al. NaGdF4 nanoparticle-based molecular probes for magnetic resonance imaging of intraperitoneal tumor xenografts in vivo. ACS Nano. 2013;7(1):330-338. 
International Journal of Nanomedicine

Dovepress

\section{Publish your work in this journal}

The International Journal of Nanomedicine is an international, peerreviewed journal focusing on the application of nanotechnology in diagnostics, therapeutics, and drug delivery systems throughout the biomedical field. This journal is indexed on PubMed Central, MedLine, CAS, SciSearch ${ }^{\circledR}$, Current Contents ${ }^{\circledR} /$ Clinical Medicine,
Journal Citation Reports/Science Edition, EMBase, Scopus and the Elsevier Bibliographic databases. The manuscript management system is completely online and includes a very quick and fair peer-review system, which is all easy to use. Visit http://www.dovepress.com/ testimonials.php to read real quotes from published authors.

Submit your manuscript here: http://www.dovepress.com/international-journal-of-nanomedicine-journal 